\title{
Screening of Allium Tester Lines in Vitro with Pyrenochaeta terrestris Filtrates
}

Andrew C. Ludwig, John F. Hubstenberger, and Gregory C. Phillips Department of Agronomy and Horticulture, New Mexico State University, Las Cruces, NM 88003-0003

\section{G. Morris Southward}

Department of Experimental Statistics, New Mexico State University, Las Cruces, NM 88003-0003

Additional index words. onion, pink-root disease

\begin{abstract}
Callus cultures were established from intraspecific lines of Allium cepa L., interspecific $F_{1}$ progeny of $A$. cepa crossed to A. fistulosum $\mathrm{L}$. and to A. galanthum $\mathrm{L}$., advanced generations of $A$. fistulosum $\mathrm{x} A$. cepa backcrossed to $A$. cepa, and lines of A. fistulosum and $A$. galanthum. These genotypes had been identified as susceptible, resistant, or partially resistant tester lines based on prior seedling and field nursery screenings using the pink-root pathogen Pyrenochaeta terrestris (Hansen) Gorenz, Walker and Larson. Tester line calli were challenged in vitro with culture filtrates of the fungal pathogen and were assessed by visible damage ratings expressed as the percentage of pigmentation in response to the filtrate. The degrees of callus sensitivity to the filtrate observed in vitro corresponded well with the in vivo tester line classifications. These results eliminated the possible confounding influence of using various species of Allium for in vitro screening. Our results indicated the suitability of the in vitro screening approach for the possible identification of useful segregants or somaclonal variants possessing pink-root resistance. However, in vivo pathogenicity may involve mechanisms in addition to sensitivity to the putative toxins present in the filtrate.
\end{abstract}

Pink-root disease results in extensive damage to the root system of common bulbing onion (Allium cepa). Pink root is considered a limiting factor in onion production (Porter and Jones, 1933). The use of resistant cultivars is recommended as the best measure to control pink-root damage to onion (Ahmed and Harrington, 1974). However, sources of resistance within the species are limited (Jones and Perry, 1956; Nichols et al., 1965), and the introgression of resistance genes using sources of resistance in related species has been difficult (Perry and Jones, 1955).

Pyrenochaeta terrestris is known to produce a complex of toxins (Gourd et al., 1988). Fungal filtrates elicited similar susceptibility responses from treated onion seedlings as did plants exposed to the pathogen in the field (Hess and Weber, 1988). However, the results of these seedling screens, using culture filtrates, were unreliable unless the filtrates were partially purified. Previously, we treated in vitro-grown plantlets, shoots, and callus

Received for publication 19 Feb. 1990. Accepted for publication 28 Aug. 1991. Journal article no. 1526 of the New Mexico State Agricultural Experiment Station. We acknowledge the advice and assistance of Joe N. Corgan, Dept. of Agronomy and Horticulture, and Donald L. Lindsey, Dept. of Entomology, Plant Pathology, and Weed Sciences. The cost of publishing this paper was defrayed in part by the payment of page charges. Under postal regulations, this paper therefore must be hereby marked advertisement solely to indicate this fact. cultures of susceptible $A$. cepa, resistant $A$. galanthum, resistant $A$. fistulosum, and resistant reciprocal interspecific $\mathrm{F}_{1}$ hybrids of A. cepa $\times$ A. fistulosum with crude culture filtrates of $P$. terrestris containing the putative toxins (Gourd et al., 1988). In vitrogrown plantlets and shoots treated with crude filtrates did not show the expected susceptible or resistant response. This result was consistent with those reported by Hess and Weber (1988) for seedling screens using crude filtrates. In contrast, onion callus treated with the crude filtrates showed the corresponding degree of whole-plant susceptibility or resistance to the pathogen, according both to ratings for visible damage to callus and fresh weight growth responses (Gourd et al., 1988). The callus culture approach showed promise as an effective genetic tool for screening and selecting pink-root-resistant onion lines. However, the possibility remained that the observed correlation in response was confounded with the various species of Allium composing the tester lines used in the previous study (Gourd et al., 1988).

We report here a more complete assessment of the in vitro callus culture screening procedure using a diverse array of interspecific and intraspecific tester lines. Both susceptible and partially resistant entries from A. cepa were included in the study to ascertain whether species differences among tester lines were confounded with the observed resistance or susceptibility during the previous evaluation of the in vitro screening procedure.
Tester lines were chosen on the basis of their in vivo responses to the pathogen (Table 1). 'Temprana' and A. fistulosum x A. cepa NM8273 also were used in the previous in vitro screening study (Gourd et al., 1988). In vivo responses of the tester lines to the pathogen were determined previously in seedling screens using controlled-temperature tanks (Del Cid, 1988; Nelson, 1987) and in field tests using pink-root nurseries (J.N. Corgan, unpublished).

Callus cultures of each tester line were established from shoot-tip explants and maintained as described by Gourd et al. (1988). Control callus was grown on a standard onion callus growth medium (Gourd et al., 1988). The P. terrestris Franzoy strain (collected and isolated from a field near Hatch, N.M., by D.L. Lindsey) used in this study was chosen because of its high expression of virulence in previous seedling screens (Nelson, 1987). Cultures of the fungus were maintained in our laboratory, and filtrates were prepared following the methodology of Gourd et al. (1988). A single batch of culture filtrate was stored at 4C and used in all experiments of this study. Treated callus was grown on a medium of the same composition, with Franzoy culture filtrate incorporated at $10 \%(\mathrm{v} / \mathrm{v})$ of the total before autoclaving. The virulence of the autoclaved culture filtrate was confirmed by following the methods of Gourd et al. (1988).

The primary screening experiment performed in this study was comprised of three repetitions. Unequal numbers of plants (sampling units) from each tester line were sampled as available (Table 1). Interspecific $\mathrm{F}_{1}$ hybrids of $A$. fistulosum x A. cepa were unique plants, limiting the sampling unit to one plant from these tester lines. Within each plant sampled, paired pieces of callus (experimental units) weighing $\approx 750 \mathrm{mg}$ were distributed randomly onto a plate $(100 \times 20$. $\mathrm{mm}$ petri dishes) of control and a plate of treatment medium. Each repetition of the experiment consisted of three replications, with one experimental unit per plate. Thus, there were six experimental units from each sampling unit in each repetition. The cultures were incubated at $25 \mathrm{C}$ with a $16-\mathrm{h}$ photoperiod $\left(15 \mu \mathrm{mol} \cdot \mathrm{m}^{-2} \cdot \mathrm{s}^{-1}\right)$.

After 30 days of incubation, visible damage to callus was recorded as a percentage of each callus (experimental unit) showing pigmentation. Data were estimated to the nearest 5\% increment, assuming a normal distribution, using a transparent grid superimposed over each callus. The grid was marked in 4-mm squares, and its height above the callus was adjusted to encompass the surface area of the callus within 20 squares. This method of assessment was based on the most promising in vitro screening procedure identified in the previous study (Gourd et al., 1988); namely, rating for visible damage to callus in response to fungal culture filtrate.

Response means were calculated for the analyses of variance using the calculated differences between the paired treated and control experimental units (calli) comprising each replication, and then averaged over the plants 
Table 1. Sensitivity of Allium tester line calli to Pyrenochaeta terrestris Franzoy filtrate incorporated into the culture medium, after 30 days of incubation.

\begin{tabular}{|c|c|c|c|c|}
\hline Allium tester line $\mathrm{z}^{\mathrm{z}}$ & $\begin{array}{l}\text { Classification } \\
\text { of plant } \\
\text { response to } \\
\text { pathogen }\end{array}$ & $\begin{array}{l}\text { No. plants } \\
\text { sampled } \\
\text { in vitro }\end{array}$ & $\begin{array}{l}\text { Visible damage } \\
\text { ( } \% \text { calli affected), } \\
\text { trcatcd - control }{ }^{x}\end{array}$ & $\begin{array}{c}\text { Probability } \\
\text { to reject HO: } \\
\text { treated }- \text { control }=0\end{array}$ \\
\hline A. fistulosum Ishikura & Resistant & 10 & $0.0 \mathrm{a}$ & NS \\
\hline A. fistulosum $\times$ A. cepa $\mathrm{F}_{1} \mathrm{NM} 8121$ & Resistant & 1 & $0.0 \mathrm{a}$ & NS \\
\hline A. galanthum PI 280666 & Resistant & 16 & $0.9 \mathrm{ab}$ & NS \\
\hline $\mathrm{BC}_{2}$ and $\mathrm{BC}_{1}-\mathrm{F}_{2} \mathrm{NM} 86-64-4^{\mathrm{w}}$ & Resistant & 10 & $1.1 \mathrm{ab}$ & NS \\
\hline A. fistulosum $\times$ A. cepa $\mathrm{F}_{1} \mathrm{NM} 8273$ & Resistant & 1 & $1.6 \mathrm{ab}$ & NS \\
\hline A. cepa NuMex Sunlite & Partial resistance & 10 & $3.6 \mathrm{bc}$ & $* *$ \\
\hline A. cepa $\times(A$. fistulosum $\times A$. cepa $)$ & & & & \\
\hline $\mathrm{BC}_{2}$ and $\mathrm{BC}_{1}-\mathrm{F}_{2} \mathrm{NM} 86-64-3^{w}$ & Resistant & 10 & $3.9 \mathrm{bc}$ & $* *$ \\
\hline A. galanthum $\times$ A. cepa $\mathrm{F}_{1}$ gac 76 & Resistant & 4 & $5.9 \mathrm{c}$ & *** \\
\hline A. cepa Texspan & Partial resistance & 7 & $9.9 \mathrm{~d}$ & $* * *$ \\
\hline A. сера NM8662 & Partial resistance & 16 & $12.0 \mathrm{~d}$ & $* * *$ \\
\hline Texspan $\times$ Temprana NM851008 & $\begin{array}{l}\text { Partial resistance } x \\
\text { susceptible }\end{array}$ & 16 & $15.6 \mathrm{e}$ & $* * *$ \\
\hline A. cepa Temprana & Susceptible & 7 & $34.9 \mathrm{f}$ & *** \\
\hline
\end{tabular}

${ }^{2}$ All materials were provided by J.N. Corgan, New Mexico State Univ., Las Cruces, except A. galanthum x A. cepa gac76 provided by G. McCollum, U.S. Dept. of Agriculture, Beltsville, Md.

${ }^{y}$ According to previous seedling screens (Del Cid, 1988; Nelson, 1987) and field nursery tests (J.N. Corgan, personal communication); partial resistance derived from intraspecific sources.

${ }^{x}$ Callus sensitivity to fungal filtrate was assessed as percentage of experimental units (calli) showing pigmentation (visible damage) estimated to the nearest 5\% increment, analyzed as the calculated difference of paired treated and control experimental units from each plant sampled and averaged over plants sampled within tester lines. Values followed by different letters are significantly different at $P=0.05$ using LSD mean separation.

"Because of the manner in which the seed cage was constructed, progeny resulting from pollination of $\mathrm{BC}_{1}$ plants by $A$. cepa and from intermating of $\mathrm{BC}_{1}$ plants could not be distinguished. Thus, it was assumed that the progeny may contain both $\mathrm{BC}_{2}$ and $\mathrm{BC}_{1}$ $\mathrm{F}_{2}$ individuals.

Ns,**,***Nonsignificant or significant at $P=0.01$ or 0.001 , respectively.

sampled within each tester line. This method of analysis accounted for inherent differences in callus response potential between plants sampled and eliminated the need for any additional covariance analysis. Data were analyzed using SAS (SAS Institute, Cary, N.C.) for a randomized complete block design.

The analysis of variance for the primary screening experiment (Table 2) indicated that tester line effects were significant. Most of the variation observed for the response means of the tester lines was associated with the plants sampled within tester lines, while there was little variation associated with the replications within plants sampled. Visible damage ratings reflected the lowest degree of callus sensitivity to the fungal filtrate among the A. fistulosum, A. galanthum, A. fistulosum $\times$ A. cepa $\mathrm{F}_{1}$, and $A$. cepa $\times$ (A. fistulosum x A. cepa) $\mathrm{BC}_{2} / \mathrm{BC}_{1}-\mathrm{F}_{2} \mathrm{NM} 86-64-4$ tester lines (Table 1). The highest degree of filtrate sensitivity was exhibited by 'Temprana' callus. The cross between 'Texspan' and 'Temprana' showed an intermediate population mean for filtrate sensitivity compared to the two parental lines. However, this population contained a mixture of two classes of individual plants that showed either a high or a low callus sensitivity to the filtrate, providing evidence that the population was segregating for susceptibility or resistance. Those tester lines showing the lowest degree of filtrate sensitivity in vitro showed a nonsignificant treatment effect. The other tester lines showed a significant treatment effect.

A formal correlation analysis was not possible due to the differences between the con- ditions of the in vitro experiments in the present study and the previous in vivo experiments. Overall, however, the degrees of callus sensititvity to fungal filtrate observed in vitro appeared to correspond well with the previous in vivo classifications of tester line susceptibility or resistance to the pathogen. The only exceptions noted were the $A$. galanthum x A. cepa $\mathrm{F}_{1}$ and $A$. cepa $\times$ (A. fistulosum $\mathrm{x}$ A. cepa) $\mathrm{BC}_{2} / \mathrm{BC}_{1}-\mathrm{F}_{2} \mathrm{NM} 86-64-3$ tester lines. These two showed a significant treatment effect in the in vitro experiment (Table 1), but previously were classified as resistant in vivo. The raw data (not shown) suggested that our sampling of these tester lines contained segregating individuals, which may account for the calli of these lines exhibiting a higher degree of sensitivity to the filtrate when response means were calculated than that predicted by previous in viva classifications. Nevertheless, these two tester lines exhibited a lower degree of callus sensitivity to the filtrate than did two of the three tester lines previously classified as partially resistant in vivo.

An alternative explanation for this result is that the higher degree of filtrate sensitivity exhibited by callus of these two tester lines than that predicted may be due to a difference in the physiology of callus cultures compared to whole plants. For example, morphological barriers to hyphal invasion present in intact plants exposed to the pathogen would not be present in callus exposed to the fungal filtrate. Callus (and plants) of these two tester lines may have a high sensitivity to the fungal toxins, but the plants may possess morphological barriers to resist hyphal invasion. Thus, a greater expression of resistance in the plants treated under the described in vivo conditions would be expected, compared to the callus treated under the described in vitro conditions. The degree of sensitivity of callus to filtrates may have a limited capability to reflect the inherent mechanisms of infection and pathogenicity in corresponding plants, apart from those mechanisms involving the action of fungal toxins.

The significant effects of experiment repetition and of tester line interaction with experiment repetition (Table 2) during the primary screening experiment may have been influenced by the increasing age of the callus lines or of the stored filtrate used, or both. A 'final experiment was conducted to test whether the age or source of the filtrate influenced the screening results. This experiment was conducted to compare stored filtrates of the Franzoy strain with fresh filtrates of the PHW-387-6 strain [obtained from C.E. Peterson (deceased), Madison, Wis.] used previously for in vitro screening (Gourd et al., 1988). The conditions of the final experiment were the same as for those described previously, except that the analysis of variance was performed for a completely randomized design with a single repetition.

The analysis of variance for visible damage ratings in the final experiment (Table 3 ) indicated that filtrates obtained from the two fungal strains, Franzoy and PHW-387-6, were equivalent in their effect on tester line callus. Each source of filtrate showed a significant treatment effect compared to the control, which lacked a fungal filtrate. The response means calculated from the data offered no new insights to the interpretation of the 
Table 2. Analysis of variance for sensitivity of Allium tester line calli to Pyrenochaeta terrestris Franzoy filtrate, assessed with visible damage ratings after 30 days of incubation.

\begin{tabular}{lrrr}
\hline \hline Source of variation & df & Mean square & F value \\
\hline Tester line (TL) & 11 & 892.62 & $104.63^{* * *}$ \\
Rep (Tester line) & 24 & 8.53 & $7.81^{* *}$ \\
Experiment repetition (ER) & 2 & 103.30 & $7.32^{* * *}$ \\
TL × ER & 22 & 96.84 & 13.22 \\
Error & 48 & & \\
\hline
\end{tabular}

******Significant at $P=0.01$ or 0.001 , respectively.

Table 3. Analyses of variance and contrast comparisons for percentage of callus pigmentation (visible damage) and callus fresh-weight growth of Allium tester lines exposed or not exposed to filtrates of Pyrenochaeta terrestris strains Franzoy and PHW-387-6 for 30 days.

\begin{tabular}{lccc}
\hline \hline $\begin{array}{l}\text { Source of variation } \\
\text { or contrast comparison }\end{array}$ & df & Mean square & F value \\
\hline \multicolumn{1}{c}{ Analysis of percent } \\
callus pigmentation & (visible damage) & \\
Filtrate & 2 & 205.8 & $4.73^{*}$ \\
PHW-387-6 vs. Franzoy & 1 & 0.8 & $0.02^{\mathrm{Ns}}$ \\
Franzoy vs. control & 1 & 292.2 & $6.72^{*}$ \\
PHW-387-6 vs. control & 1 & 324.6 & $7.46^{* *}$ \\
Error & 96 & 43.5 & \\
& Analysis of callus fresh wt growth & $0.52^{\mathrm{Ns}}$ \\
Filtrate & 2 & 66344 & $0.07^{\mathrm{Ns}}$ \\
PHW-387-6 vs. Franzoy & 1 & 8452 & $0.53^{\mathrm{Ns}}$ \\
Franzoy vs. control & 1 & 67226 & $0.96^{\mathrm{Ns}}$ \\
PHW-387-6 vs. control & 1 & 123354 & 127887 \\
Error & 96 & & \\
\hline
\end{tabular}

Ns $* * *$ Nonsignificant or significant at $P=0.05$ or 0.01 , respectively.

analysis of variance and, thus, are not shown. The results of this experiment indicated that the source of filtrate was not significant. In contrast, previous studies using these two fungal strains showed significant differences in their virulence with seedlings under in vivo conditions (Nelson, 1987). The results of the current study provide evidence that the two strains have similar capacities to produce toxins, as assessed by their effectiveness in the in vitro experiment. However, the differential virulence noted under in vivo conditions may be caused in part by mechanisms of pathogenicity other than the production of toxins.

Another protocol of the in vitro screening procedure of Gourd et al. (1988) that was promising as a means to distinguish among tester lines was the use of fresh-weight growth of control callus compared to paired callus pieces treated with fungal filtrates. This method of filtrate response measurement also was evaluated in the final experiment of the present study. The analysis of variance for this response variable indicated there was no significant effect by either of the two sources of filtrate on callus growth in this experiment
(Table 3). Response means did not facilitate interpretation of the analysis of variance and are not shown. Treated calli exhibited growth rates of $75 \%$ to $80 \%$ of the growth of the paired control callus pieces within most tester lines. However, these responses apparently were confounded by inherent differences in growth or response potential of the plants sampled within and among tester lines. This result did not agree with the results of Gourd et al. (1988). However, in the previous case only a few plants were sampled within a few tester lines, whereas we sampled more plants within most tester lines; also, a more diverse array of interspecific and intraspecific tester lines was represented. The results of the more extensive survey conducted in this study indicated that growth rate differences between paired treated and control calli did not adequately distinguish between Allium tester lines challenged with $P$. terrestris filtrates.

In conclusion, the present experiments evaluated 12 tester lines from diverse sources of Allium, grown as callus cultures and challenged with Pyrenochaetu filtrates. Assessments of visible damage to the callus successfully distinguished the tester lines ac- cording to their degree of sensitivity to the filtrate. Tester line sensitivity to the filtrate in vitro corresponded well with in vivo classifications of tester line susceptibility or resistance to the pathogen using this procedure. However, some evidence suggested in vivo pathogenicity may involve mechanisms in addition to sensitivity to the putative toxins contained in the filtrate. Nevertheless, this in vitro screening approach should be a useful tool to identify valuable segregants in a breeding program aimed at improving resistance to onion pink-root disease. This procedure also could be adapted and applied for the recovery of somaclonal variants that would have potential value in a breeding program, following the regeneration of plants (Phillips and Hubstenberger, 1987) from callus challenged with the fungal filtrate.

\section{Literature Cited}

Ahmed, A.A.E.G. and J.F. Harrington. 1974. Onion seed yield as affected by pink root disease, soil fumigation, mother bulb fertilization and bulb size. HortScience 9:394-396.

Del Cid, A.R. 1988. Allium species and cultivar screening for pink root resistance across isolates and inoculum levels. MS Thesis, New Mexico State Univ., Las Cruces.

Gourd, J.M., G.M. Southward, and G.C. Phillips. 1988. Response of Allium tissue cultures to filtrates of Pyrenochaetu terrestris. HortScience 23:766-768.

Hess, W.M. and D.J. Weber. 1988. Assays for determining resistance and susceptibility of onion cultivars to the pink root disease. Phytopathology 78:115-117.

Jones, H.A. and B.A. Perry. 1956. Inheritance of resistance to pink root in onions. J. Hered. 47:3334.

Nelson, S.R. 1987. Interaction of temperature, isolate and cultivar on pink-root of onion. MS Thesis, New Mexico State Univ., Las Cruces.

Nichols, C.G., W.H. Gabelman, R.H. Larson, and J.C. Walker. 1965. The expression and inheritance of resistance to pink root in onion seedlings. Phytopathology 55:752-756.

Perry, B.A. and H.A. Jones. 1955. Performance of short-day pink-root-resistant varieties of onions in southern Texas. Proc. Amer. Soc. Hort. Sci. 66:350-353.

Phillips, G.C. and J.F. Hubstenberger. 1987. Plant regeneration in vitro of selected Allium species and interspecific hybrids. HortScience 22:124125.

Porter, D.R. and H.A. Jones. 1933. Resistance of some of the cultivated species of Allium to pink root (Phoma terrestris). Phytopathology 23:290298. 